\title{
Was Feuerbach right: are we what we eat?
}

\author{
Giovanni Cizza1 and Kristina I. Rother²
}

\author{
${ }^{1}$ Section on Neuroendocrinology of Obesity and ${ }^{2}$ Section on Pediatric Diabetes and Metabolism, NIDDK, NIH, Bethesda, Maryland, USA.
}

\begin{abstract}
Food and stress are powerful modulators of the body-mind connection, which is imbalanced in obese individuals. Why do we choose chocolate over an apple when overworked and stressed, and why does comfort food make us feel better? Two independent studies in the JCI, one in this issue, home in on the role of stress on gut hormones and food choices and, conversely, on the effect of the intestinal system on modulation of brain activity by sadness. These studies broaden our understanding of the ties between food and mood and underscore promising targets for obesity treatments.
\end{abstract}

\section{Introduction}

The concept of homeostasis, i.e., the constancy of the inner environment or "internal milieu," was originally formulated by the French physiologist Claude Bernard (1). He postulated that the fixity of the milieu depends on a perfect dynamic balance of the organism, such that external variations are promptly equilibrated. This is the case in normal-weight individuals, who achieve weight maintenance despite occasional 5,000 kcal Thanksgiving dinners and strenuous hiking trips interrupted only by a short rest and a meager sandwich. How is this weight balance achieved? An elaborate system of signals from within the body - specifically the gastrointestinal tract and the adipose tissue, which form key parts of this interoceptive system that underlies weight homeostasis - and from outside the body (which are conveyed by the exteroceptive system) constantly keep the brain informed about its nutritional status. The brain, in turn, conveys feedback to the periphery in order to counterbalance over- or underfeeding.

The interoceptive system sends afferent inputs via parasympathetic (vagal and glossopharyngeal) afferents to the nucleus tractus solitarius and via small-diameter primary sympathetic fibers to relay stations in the spine, medulla, and higher nervous system centers. These sympathetic inputs contain information about thermal, chemical, mechanical, metabolic, and hormonal changes and originate in skin, muscle, joints, and viscera. Among the hormonal signals, the enteroendocrine system plays a special role, which is the focus of

Conflict of interest: The authors have declared that no conflict of interest exists.

Citation for this article: J Clin Invest. 2011; 121(8):2969-2971. doi:10.1172/JCI58595. two studies reported in the JCI (Figure 1) $(2,3)$. Specifically, Van Oudenhove's clinical (2) and Chuang's preclinical (3) studies assess how food modulates sadness and how stress and sadness modulate hunger and food preferences.

\section{Sadness modulates gut-brain messages}

The effects of consciously ingested fat have been extensively studied, and it is well known that comfort food stimulates our internal reward system (4). The observation by Van Oudenhove et al. that food intake without awareness (i.e., without visual, taste, and olfactory inputs) can modify emotions (2) is novel and interesting. This study tested the effects of an intragastric infusion of a fatty acid solution or saline in healthy, nonobese individuals in a blinded fashion. Subjects were then presented with sad or neutral classical music coupled with images of consonant emotional meaning, and their responses were assessed by neuroimaging. When subjects were pretreated with a fatty acid solution, their responses to experimentally induced sadness were attenuated. Conversely, being presented with sad music or pictures diminished the sense of fullness associated with fat ingestion. The size of the effect, reducing the intensity of sad emotions by almost half, may be clinically meaningful, since it is in the order of magnitude of pharmacological effects of antidepressants on mood scores.

The work of Van Oudenhove and colleagues (2) raises several questions. First, what are the endocrine and behavioral responses (i.e., modulation of sadness) to other macronutrients (carbohydrates, protein, or a mixture thereof)? Second, how are other emotions and sentiments (e.g., empathy, anger, and anxiety) affected by fat and other macronutrients? Third, is there a dose response of the fat load on emotion in healthy individuals, and is this response altered in varied nutritional states (e.g., obesity and anorexia nervosa)?

Despite the clear physiologic relevance of the work of Van Oudenhove and colleagues (2), some limitations exist. These include its descriptive nature and small number of participants. In particular, one wonders which neural and endocrine mechanisms mediate the attenuation of sadness. Since the fatty acid solution was administered directly to the stomach, likely mechanisms include the peptide hormone ghrelin, which is produced predominantly by distinct ghrelin-producing cells in the stomach and other parts of the gastrointestinal tract, as well as stimulation of parasympathetic terminals located in the stomach. The latter would implicate a function for the gut peptide cholecystokinin (CCK), which has previously been shown to have a crucial role in mediating the effects of intragastric fatty acid solutions on brain activity (5). Additional support for a role for CCK is provided by the fact that phylogenetically, receptors for CCK are known not only for their role in digestion, but also for roles in memory function and learning and in modulation of panic and anxiety (6). Furthermore, gender-related differences were not addressed, and the absence of an obese study cohort leaves the most important question unanswered: Do obese individuals experience a similar anti-sadness effect after fat ingestion? Finally, because all experiments were performed in the morning, an effect of the circadian rhythm was not addressed. Of note, individuals who are chronically sleep deprived tend to ingest comfort food in the late evening, and night eating syndrome is an emerging, albeit controversial, eating disorder.

\section{Stress and ghrelin}

Similar to the work of Van Oudenhove et al. in humans (2), Chuang et al. investigated in mice the effects of an externally induced state of mind (3), in their case, stress. This is certainly relevant, since increasing levels of stress are thought to be key influences on the obesity epidemic in humans in addition to the "Big Two" 


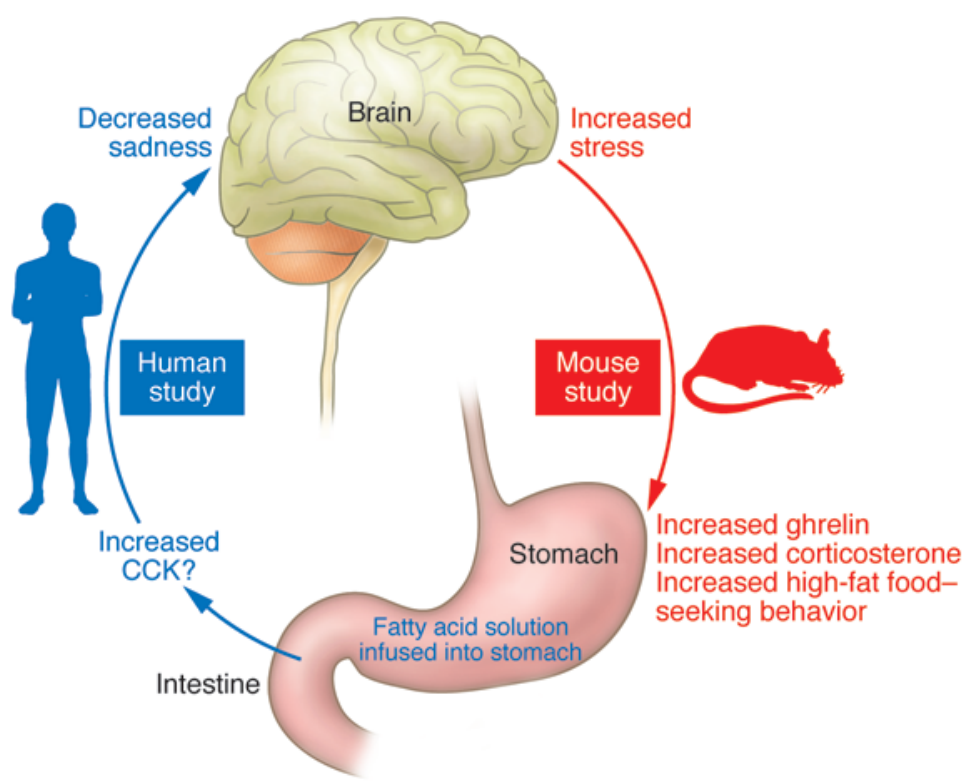

(too much food and too little exercise). Chuang and colleagues used a model of psychosocial stress to investigate its effects on feeding behavior (3). For several days, male mice were exposed to bouts of social subordination by an older aggressor, a model of chronic social defeat stress (CSDS). This procedure resulted in robust increases in acyl-ghrelin, the active circulating form of ghrelin, and, as expected, the stress hormone corticosterone. In addition, these animals became behaviorally inhibited and spent more time in corners and less time exploring. Chuang et al. coupled CSDS with conditioned place preference, in which the animals were restricted on odd days to one side of the chamber, which contained high-fat food, and on even days to the other side of the chamber, where regular chow was accessible. Socially stressed animals ate more high-fat food and spent more time on the side of the chamber where the high-fat food had originally been placed. Chuang et al. provided evidence that these behaviors were ghrelin dependent: time spent exploring was attenuated, and time spent hiding in corners reciprocally increased in mice lacking ghrelin receptors. In addition, these behaviors were rescued by expression of ghrelin receptors in selected areas of the brain, such as the arcuate nucleus and the ventral tegmental area, which contain catecholaminergic neurons expressing tyrosine hydroxylase, the rate-limiting step of catecholamine biosynthesis. These brain regions are involved in reward behavior and mood regulation.
The study by Chuang et al. (3), with its focus on ghrelin, leads us to wonder whether ghrelin, in addition to its known function as an appetite stimulant, may also play a role in withstanding stress, especially psychosocial stress, a common situation in everyday life. A stress-induced rise in ghrelin levels may facilitate coping mechanisms. Ghrelin has potent antiinflammatory effects mediated via inhibition of proinflammatory cytokines (7). It also acts as a neuropeptide: sleep deprivation increases ghrelin (8), whereas ghrelin administration promotes restorative slow-wave sleep (9); ghrelin is therefore considered a sleep-promoting factor. Furthermore, ghrelin may have antinociceptive functions (10) and facilitate learning behavior and memory tasks (11). Thus, a rise in ghrelin levels may aid in withstanding pain, lack of sleep, and subclinical inflammation, all of which are associated with stress.

Clinically, these effects of ghrelin may be especially relevant to patients undergoing bariatric surgery (12). In sleeve gastrectomy, a large portion of the stomach is removed, and thus the main source of ghrelin-producing cells is acutely eliminated. Are postoperative patients less able to cope with stress? Most likely, the answer is no. In the long run, ghrelin levels recover after surgery, and it has recently been shown in an animal model of bariatric surgery that the observed major changes in ghrelin occur only in total ghrelin, not in active ghrelin (13).

The work of Chuang and colleagues (3) provides a highly plausible mechanistic explanation as to why stress induces indi-

\section{Figure 1}

The body-mind connection: how emotions modulate food intake, and how food modulates emotions. Left: Van Oudenhove et al. show in humans that gastric infusion of a fatty acid solution decreases experimentally induced sadness, as indicated by visual analog scores and neuroimaging (2). The underlying mechanisms may in part depend on CCK, as shown by a previous study (5). Right: Chuang et al. report that psychosocial stress in mice increases levels of ghrelin and corticosterone, while stimulating high-fat food-seeking behavior (3). Taken together, the results of these two studies underline the importance of bidirectional interactions between the gastrointestinal system and the brain. viduals to eat calorically dense comfort foods. The logical next step is to determine whether this study in male mice relates to men and, furthermore, whether it holds true in both males and females. The stress model used, the intruder paradigm and its consequences of social subordination, is definitely relevant to male rats and likely to humans. Given that the way in which social hierarchy is established and maintained displays sexual dimorphism across species, this stress model may not be relevant for the female gender. Furthermore, the ultimate question of how stress raises ghrelin levels remains to be answered.

\section{Is it what we eat, or what's eating us?}

Van Oudenhove et al. (2) and Chuang et al. (3) investigated the connections among psychosocial stress, sadness, and food intake; therefore, their data are relevant to the obesity epidemic. Clinicians, health administrators, politicians, and affected individuals have a frustrating lack of effective tools to alleviate obesity. In our opinion, this frustration can be accounted for by several factors. First, the obesity epidemic is a multifactorial phenomenon with clear environmental and genetic components. Thus, at a societal level, it will not be defeated by pharmacological (drugs) or surgical (bariatric surgery) approaches operating in a vacuum. Second, our modern environment is characterized by unlimited food and limited need for physical activity. This unprecedented situation has found us ill-equipped in evolutionary terms: body weight changes are defended in an asymmetrical fashion, as 
we are better at gaining weight than losing it. Finally, our understanding of the mechanisms controlling appetite and energy balance remains incomplete. Brain plasticity and the redundancy of appetite-regulating circuits defeat single-target therapeutic approaches. As a consequence, no new pharmacological obesity blockbuster drug seems to be on the horizon.

It was in the context of the stress and turmoil of the German revolution in 1848 that the German philosopher Ludwig Feuerbach used his famous phrase, "We are what we eat" (14). He further stated, "A man who enjoys only a vegetable diet is only a vegetating being...." Although we don't agree with the latter statement, we agree with his vision of connecting human essence, stress, and nutrition. Indicators of stress, whether antidepressant use, anxiety level, or completed or attempted suicides, are on the rise in industrialized countries in parallel with the obesity epidemic $(15,16)$. Obesity is more prevalent in the lower socioeconomic classes, where levels of social subordination are higher. Like obesity, sleep deprivation is also more common among the less privileged (17). It is therefore tempting to attribute our bulging waistlines to an attraction to comfort foods that alleviate stress and to deficient restorative sleep. The picture is obviously more complex and likely to involve individual differences in coping with stress. Some of these differences may be rooted in our genome, others in our cultural environment and the value each culture attributes to food or sleep, and yet others in our temperament and personality.

The field of research on the mind-body connection has suffered from a Cartesian top-down approach, in which the brain or mind is presumed to influence the body. However, the intricate relationship of body and mind is egalitarian in nature and bidirectional: the body is capable of powerfully modulating our emotions, as shown by both Van Oudenhove et al. (2) and Chuang et al. (3), and as witnessed by physicians every day. An example is the neonatologist who gives some sugar to a neonate before performing an invasive procedure to shorten the time the baby cries in pain (18).

In a social species, like humans, the survival instinct of the individual has to be balanced with the survival instinct of the species. Sadness is a universal emotion, with common facial expressions equally recognizable by completely different cul- tures. Conversely, the inability to express emotions is a feature of serious mental conditions such as autism (19). Appetite, which is stimulated by ghrelin, is essential for survival of the individual; empathic sadness, a uniquely human sentiment, may be detrimental to the survival of the individual, but it is definitely instrumental to the survival of the species. Sadness, depressed mood, and the like are often regarded by our hedonistic society as negative emotions. Antidepressants and other drugs are subliminally, or even openly, advertised as cosmetic neuropharmacological tools. Before indiscriminately engaging in mood-manipulating techniques, whether swallowing a pill or eating a hamburger, we need to interrogate ourselves on the evolutionary origins of emotions.

\section{Conclusions}

The reports from Van Oudenhove et al. (2) and Chuang et al. (3) illustrate that the history of endocrinology has come full circle. Enteroendocrine hormones were the first hormones to be discovered and have regained great importance (e.g., glucagon-like peptide 1 , gastric inhibitory polypeptide, CCK, and ghrelin). In June 1905, Ernest Starling announced the discovery of the first hormone, named secretin, to the Royal College of Physicians in London (20); the discovery of gastrin followed shortly thereafter. About 50 years earlier, Feuerbach had exclaimed, "We are what we eat," a statement made in the context of malnutrition (14). While he denounced a reference to his quote as "scurrilous expression of modern sensualistic pseudo-wisdom" a few years later, we dare to object. Today, its continued relevance is indeed supported by sophisticated neuroimaging and molecular biology. Approaches to treating obesity should certainly take the link between stress and food into account; the lever should be inserted at the origin of stress.

\section{Acknowledgments}

We thank Rebecca Brown (NIDDK, NIH) for her constructive comments. This work was supported by the Intramural Program of the NIH, NIDDK.

Address correspondence to: Giovanni Cizza, Building 10, CRC, Rm. 6-3940, Bethesda, Maryland 20892-1613, USA. Phone: 301.496.8711; Fax: 301.480.2047; E-mail: cizzag@intra.niddk.nih.gov.
1. Noble D. Claude Bernard, the first systems biologist, and the future of physiology. Exp Physiol. 2008;93(1):16-26.

2. Van Oudenhove L, et al. Fatty acid-induced gutbrain signaling attenuates neural and behavioral effects of sad emotion in humans. J Clin Invest. 2011;121(8):3094-3099.

3. Chuang J-C, et al. Ghrelin mediates stress-induced food-reward behavior in mice. J Clin Invest. 2011;121(7):2684-2692.

4. Zheng H, Lenard NR, Shin AC, Berthoud HR. Appetite control and energy balance regulation in the modern world: reward-driven brain overrides repletion signals [review]. Int J Obes (Lond). 2009;33(Suppl 2):S8-S13.

5. Lassman DJ, et al. Defining the role of cholecystokinin in the lipid-induced human brain activation matrix. Gastroenterology. 2010;138(4):1514-1524.

6. Staljanssens D, et al. The CCK(-like) receptor in the animal kingdom: functions, evolution and structures. Peptides. 2011;32(3):607-619.

7. Baatar D, Patel K, Taub DD. The effects of ghrelin on inflammation and the immune system. Mol Cell Endocrinol. 2011;340(1):44-58.

8. Spiegel K, Tasali E, Penev P, Van Cauter E. Sleep curtailment in healthy young men is associated with decreased leptin levels, elevated ghrelin levels, and increased hunger and appetite. Ann Intern Med. 2004;141(11):846-850.

9. Weikel JC, et al. Ghrelin promotes slow-wave sleep in humans. Am J Physiol Endocrinol Metab. 2003;284(2):E407-E415

10. Guneli E, Gumustekin M, Ates M. Possible involvement of ghrelin on pain threshold in obesity. Med Hypotheses. 2010;74(3):452-454.

11. Cuellar JN, Isokawa M. Ghrelin-induced activation of cAMP signal transduction and its negative regulation by endocannabinoids in the hippocampus. Neuropharmacology. 2011;60(6):842-851.

12. Nandagopal R, Brown RJ, Rother KI. Resolution of type 2 diabetes following bariatric surgery: implications for adults and adolescents [review]. Diabetes Technol Ther. 2010;12(8):671-677.

13. Patrikakos P, et al. Long-term plasma ghrelin and leptin modulation after sleeve gastrectomy in Wistar rats in comparison with gastric tissue ghrelin expression [published online ahead of print]. Obes Surg. doi:10.1007/s11695-011-0426-x.

14. Cherno M. Feuerbach's "Man is what he eats": a rectification. J Hist Ideas. 1963;24(3):397-406.

15. Siervo M, Wells JC, Cizza G. The contribution of psychosocial stress to the obesity epidemic: an evolutionary approach. Horm Metab Res. 2009; 41(4):261-270.

16. Rowshan Ravan A, Bengtsson C, Lissner L, Lapidus L, Björkelund C. Thirty-six-year secular trends in sleep duration and sleep satisfaction, and associations with mental stress and socioeconomic factors--results of the Population Study of Women in Gothenburg, Sweden. J Sleep Res. 2010;19(3):496-503.

17. Patel NP, Grandner MA, Xie D, Branas CC, Gooneratne N. "Sleep disparity" in the population: poor sleep quality is strongly associated with poverty and ethnicity. BMC Public Health. 2010;10:475.

18. Harrison D, Bueno M, Yamada J, Adams-Webber T, Stevens B. Analgesic effects of sweet-tasting solutions for infants: current state of equipoise. Pediatrics. 2010;126(5):894-902.

19. Losh M, et al. Neuropsychological profile of autism and the broad autism phenotype. Arch Gen Psychiatry. 2009;66(5):518-526.

20. Henriksen JH, de Muckadell OB. Secretin, its discovery, and the introduction of the hormone concept. Scand J Clin Lab Invest. 2000; 60(6):463-471. 